\title{
TRANSPLANTE DE FÍGADO POR FALÊNCIA HEPÁTICA FULMINANTE SECUNDÁRIA A TRATAMENTO DE HANSENÍASE: RELATO DE CASO
}

\author{
Liver transplantation for acute liver failure following leprosy treatment: Case Report
}

\author{
Paulo Sérgio Vieira de Melo', Gabriel Guerra Cordeiro², Lucas Michael Silva Loureiro³, Pedro Renan de Melo Magalhães ${ }^{3}$, \\ Anderson André Pantoja Dias', Bernardo Times de Carvalho', Priscylla Jennie Monteiro Rabêlo', Lara Neves Souza1, \\ Olival Cirilo Lucena da Fonseca Neto', Helry Lopes Cândido', Renata Ferreira Bezerra', Cláudio Moura Lacerda ${ }^{1}$
}

\section{RESUMO}

A incidência de falência hepática fulminante de etiologia medicamentosa tem aumentado. Entre as drogas envolvidas, encontram-se antibióticos, como rifampicina e dapsona, envolvidos no tratamento da hanseníase. A síndrome de hipersensibilidade à dapsona (SHD) é uma reação adversa rara, caracterizada por febre, dermatite esfoliativa, linfadenopatia, anemia e acometimento hepático, que inclui necrose de hepatócitos e colestase. A falência hepática aguda decorrente da SHD pode ser indicação de transplante de fígado emergencial, condição pouco relatada na literatura. Foram analisados dados pré e pós-operatórios do paciente, com apoio na bibliografia atual, através de busca não sistematizada nas plataformas PubMed e Scielo. Paciente do sexo masculino, 23 anos, admitido em serviço de referência, após realizar terapia combinada para hanseníase virchowiana (dapsona, rifampicina, clofazimina), por cerca de 30 dias, evoluiu com quadro grave de icterícia progressiva, anemia, coagulopatia e encefalopatia grau IV (critérios de West-Haven), além de aumento importante de enzimas hepáticas e lesão renal aguda. O paciente foi listado para transplante hepático, com escore MELD (model for end-stage liver disease) 40, realizando-se a cirurgia pela técnica piggyback, enxerto de doador falecido. A melhora clínica foi substancial, após o procedimento. Avaliação histopatológica do fígado explantado evidenciou sinais compatíveis com quadro agudo de hepatite medicamentosa. Apesar de raramente indicado em casos de hepatite fulminante associada à SHD, o transplante hepático tem se mostrado eficaz e possibilitado melhor prognóstico aos pacientes terminais. O presente caso alerta para a necessidade de acompanhamento clínico e laboratorial de pacientes em tratamento para hanseníase, além de orientá-los em relação aos sinais e sintomas da SHD, tendo em vista suas repercussões potencialmente fatais.

Descritores: Falência Hepática Aguda, Hepatite Induzida por Medicamentos, Dapsona, Hanseníase, Transplante de Fígado.

Instituições:

1 Unidade de Transplante de Fígado, Hospital Universitário Oswaldo Cruz - Universidade de Pernambuco - Recife/PE

${ }^{2}$ Centro de Ciências Médicas, Hospital das Clínicas - Universidade Federal de Pernambuco - Recife/PE

${ }^{3}$ Curso de Medicina, Hospital Universitário Oswaldo Cruz Universidade de Pernambuco - Recife/PE

\section{Correspondência:}

Gabriel Guerra Cordeiro

Rua Frei Jaboatão, 180, Torre, CEP:50710-030, Recife/PE

Tel.: (81) 99780-1227

Email: gguerra.2607@gmail.com

Recebido em: 04/05/2019

Aceito em: 30/05/2019

\section{INTRODUÇÃO}

A hanseníase é uma doença granulomatosa crônica, que se manifesta, principalmente, em pele e nervos periféricos e apresenta alto potencial de incapacidade, representando um impacto populacional significativo, sobretudo nos países em desenvolvimento. ${ }^{1}$ Em 2017, Índia, Brasil e Indonésia constituíram $80,2 \%$ dos casos relatados em todo o mundo. Nas Américas, os casos registrados no Brasil corresponderam a 92,3\%, sendo incluído na lista dos 22 países prioritários para ações de controle da Hanseníase. 
Comparativamente, em 1985, a prevalência da hanseníase global estava em cerca de 4 milhões, enquanto dados do último registro epidemiológico da OMS, em 2018, registraram aproximadamente 200.000 indivíduos acometidos. ${ }^{2}$ Tais valores refletem o avanço das medidas terapêuticas e preventivas para essa doença, que, entretanto, se aplicam de forma desigual e levam à concentração de casos em certos países, os quais necessitam de maior atenção.

Com a maior viabilização do tratamento efetivo, através da terapia multidrogas (TMD), que associa as drogas dapsona, rifampicina e clofazimina, tem surgido um aumento expressivo nos efeitos colaterais das medicações. Os sinais adversos durante a TMD surgem em cerca de $45 \%$ dos pacientes, os quais parecem estar mais relacionados, sobretudo, à dapsona, de forma isolada, e podem resultar na interrupção do tratamento em diversos casos. ${ }^{3,4}$ A síndrome de hipersensibilidade à dapsona (SHD), apesar de pouco comum, representa uma das principais causas de morte de indivíduos que realizam TMD para hanseníase. Trata-se de uma complicação grave e rara, secundária à utilização da droga, ocorrendo entre $0,5 \%$ e $3 \%$ dos pacientes, surgindo tipicamente quatro a seis semanas após o início do tratamento. ${ }^{5,6}$ As reações idiossincráticas de hipersensibilidade à dapsona podem cursar com febre, eosinofilia, anemia, manifestações cutâneas e acometimentos sistêmicos. Em casos mais graves, pode ocorrer falência hepática fulminante (FHF), com necrose hepatocitária e colestase..$^{7,8}$ Os mecanismos subjacentes e os fatores de risco para a reação em múltiplos órgãos, associados ao uso da dapsona ainda não são claros; acredita-se que seja pela ativação do sistema imune e das citocinas inflamatórias. ${ }^{6}$

Casos de FHF levam a expressivas alterações da função hepática, de forma aguda, sendo o diagnóstico breve e o manejo adequado determinantes para a sobrevida do paciente. Nesse contexto, o transplante ortotópico de fígado (TOF), indicado em cerca de $50 \%$ dos quadros, tem se mostrado uma alternativa terapêutica eficaz, com melhora substancial da sobrevida, de $16 \%$ para $62 \%$, em pacientes com diagnóstico de FHF bem estabelecido e sem resposta ao tratamento convencional. ${ }^{9}$ Eventos de necrose hepática aguda que necessitam de transplante hepático emergencial, em pacientes secundários ao uso da dapsona, são raros na literatura. ${ }^{10} \mathrm{O}$ presente artigo objetiva descrever um caso de hepatite fulminante secundária à SHD, tratado de maneira segura e eficaz, através do transplante hepático, bem como demonstrar a importância, sobretudo nas regiões com alta prevalência de hanseníase, para essa grave e pouco abordada reação causada pela droga.

\section{RELATO DO CASO}

Paciente do sexo masculino, 23 anos, procedente de região endêmica de hanseníase e índice de massa corpórea: 26,1. Admitido em serviço de referência em hepatologia e transplante hepático, após realizar, por cerca de 30 dias, terapia combinada para hanseníase virchowiana (dapsona, rifampicina, clofazimina). Apresentava quadro grave de colestase e icterícia progressiva (Bilirrubina total e direta, respectivamente: 32,5 e $27,2 \mathrm{mg} / \mathrm{dL}$ ); anemia severa (Hemoglobina $6,9 \mathrm{~g} /$ dL e Hematócrito 21,1\%); coagulopatia com alargamento do INR $(2,58)$; e encefalopatia grau IV (critérios de West-Haven); além de aumento importante das enzimas hepáticas: aspartato aminotransferase (AST: $1332 \mathrm{U} / \mathrm{L}$ ), alanina aminotransferase (1577 U/L), e lesão renal aguda, com aumento expressivo de ureia (131 mg/dL) e creatinina $(3,4 \mathrm{mg} / \mathrm{dL})$. Ao exame clínico: estado geral grave, inconsciente (escala de coma de Glasgow: 8), icterícia intensa $(4+/ 4+)$, hipocorado, intubado, ascite volumosa (sinal de piparote presente), hepatomegalia e edema moderado de membros inferiores. O paciente preencheu os critérios do King's College para insuficiência hepática aguda e, sem resposta ao tratamento clínico, foi listado com priorização para transplante hepático emergencial com escore MELD (Model for End-stage Liver Disease) 42.

Realizou-se a intervenção cirúrgica, três dias após o encaminhamento ao serviço, através da técnica piggyback, enxerto de doador falecido, com boa perfusão e produção de bile no intraoperatório, seguido de melhora clínica substancial no seguimento póstransplante (Tabela 1). Análise histopatológica do fígado nativo evidenciou extensas áreas de necrose associadas a infiltrado inflamatório, colestase intracanalicular e alterações regenerativas dos hepatócitos, sendo compatível com quadro agudo de hepatite fulminante de natureza medicamentosa (Figuras 1 - 3).

Figura 1 - Fígado nativo removido no transplante apresentando extensas áreas de necrose de hepatócitos (áreas claras) alternandose com áreas contendo hepatócitos viáveis (áreas cor-de-rosa). VC, veia centrolobular; EP, espaço portal. $H E, 40 x$

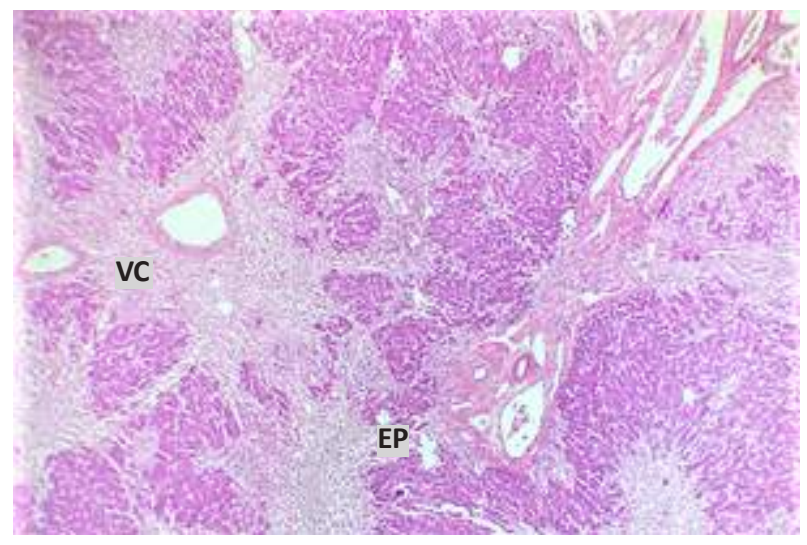


Paulo Sérgio Vieira de Melo, Gabriel Guerra Cordeiro, Lucas Michael Silva Loureiro, Pedro Renan de Melo Magalhães,

Anderson André Pantoja Dias, Bernardo Times de Carvalho, Priscylla Jennie Monteiro Rabêlo, Lara Neves Souza, Cláudio Moura Lacerda

Figura 2 - Fígado apresentando colestase intracanalicular (seta), infiltrado inflamatório linfomononuclear, áreas com extravasamento de hemácias e histiócitos contendo bile (lado esquerdo da figura). Os hepatócitos remanescentes apresentam alterações regenerativas (inferior direito). $H E, 400 x$

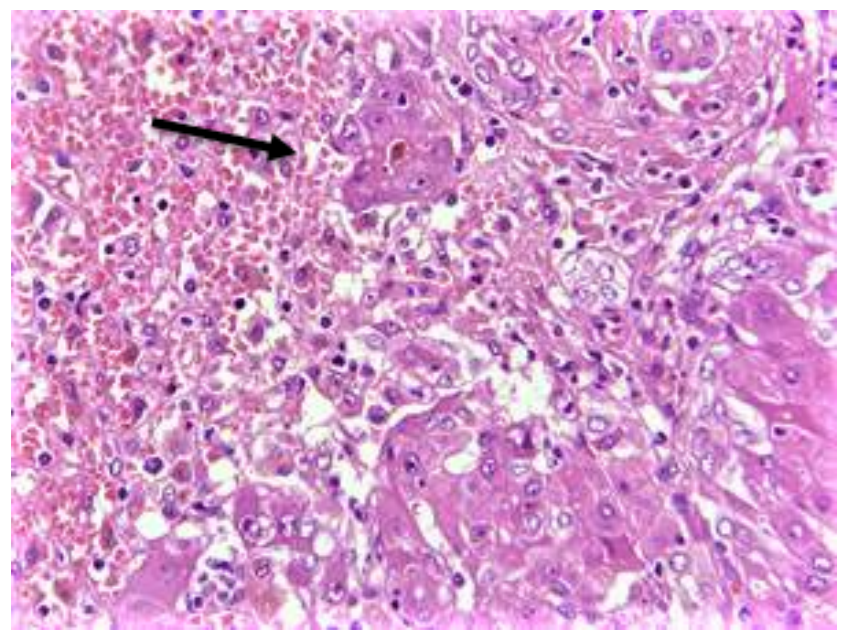

Figura 3 - Fígado apresentando corpo acidófilo (seta), infiltrado inflamatório linfomononuclear (mais intenso à direita), áreas com extravasamento de hemácias e histiócitos contendo bile (lado esquerdo). Os hepatócitos remanescentes apresentam alterações regenerativas (inferior). $H E, 400 x$

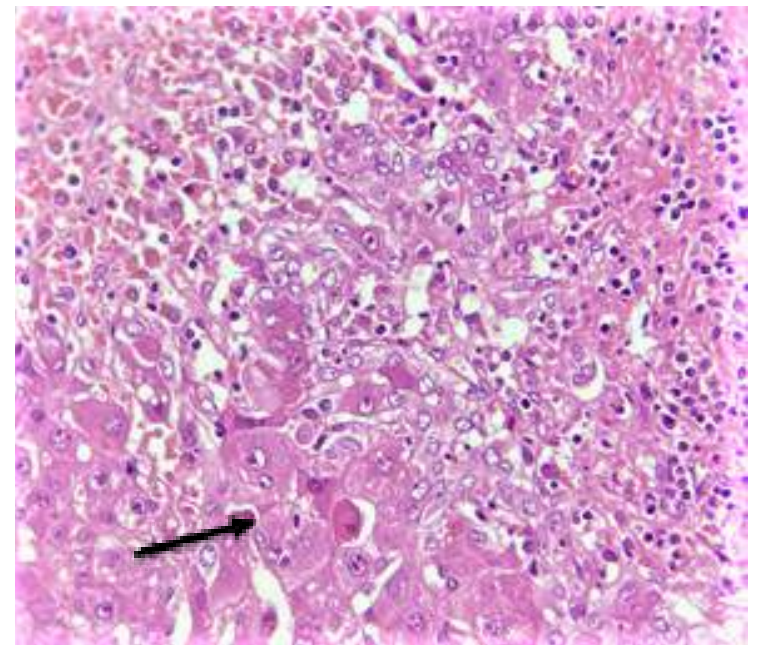

Tabela 1 - Evolução laboratorial do paciente, desde a admissão no serviço ao seguimento pós-transplante, indicando melhora importante do quadro, sobretudo na função hepática

\begin{tabular}{|c|c|c|c|c|c|c|c|c|c|c|}
\hline & BT & BD & INR & AST & ALT & DHL & UR & CRT & FBN & PQT \\
\hline Admissão no Serviço & 32,5 & 27,2 & 2,58 & 1332 & 1577 & 1195 & 131 & 3,4 & 139 & 94 \\
\hline Pré-operatório & 32,3 & 30,6 & 1,8 & 760 & 984 & 754 & 124 & 4,3 & 128 & 78 \\
\hline POI & 23 & 21,6 & 2,7 & 1616 & 1260 & 1378 & 99 & 3,8 & 113 & 60 \\
\hline 19DPO & 13,5 & 13,2 & 1,53 & 1424 & 1130 & 1237 & 98 & 3,6 & 177 & 75 \\
\hline 29DPO & 10 & 9,7 & 1,21 & 520 & 770 & 663 & 103 & 3,5 & 271 & 83 \\
\hline 4DPO & 9,1 & 8,3 & 1,04 & 266 & 538 & 658 & 81 & 3,2 & 302 & 86 \\
\hline 69DPO & 7,2 & 6,9 & 0,94 & 198 & 400 & 537 & 78 & 2,8 & 326 & 71 \\
\hline 8DPO & 6,3 & 5,7 & 0,98 & 245 & 383 & 424 & 82 & 2,8 & 288 & 81 \\
\hline 10DPO & 5,1 & 4,8 & 0,95 & 123 & 207 & 408 & 73 & 2,8 & 315 & 90 \\
\hline 159DPO & 3,6 & 3,4 & 1,0 & 77 & 57 & 261 & 85 & 2,7 & 425 & 104 \\
\hline 20DPO & 1,7 & 1,5 & 0,96 & 48 & 41 & 197 & 80 & 2,6 & 449 & 131 \\
\hline 25DPO & 0,8 & 0,6 & 1,02 & 42 & 31 & 104 & 67 & 2,5 & 414 & 146 \\
\hline
\end{tabular}

POI: Pós-operatório Imediato; DPO: Dia Pós-operatório; BT: Bilirrubina Total; BD: Bilirrubina Direta; INR: Índice Internacional Normalizado; AST: Aspartato Aminotransferase; ALT: Alanina Aminotransferase; DHL: Desidrogenase Láctica; UR: Ureia; CRT: Creatinina; FBN: Fibrinogênio; PQT: Contagem de Plaquetas (103).

\section{DISCUSSÃO}

FHF é um quadro complexo e grave, que provoca rápido declínio da função hepática (Índice Internacional Normalizado - INR $\geq 1,5$ ) e desenvolvimento de encefalopatia, até 26 semanas após o aparecimento de icterícia, em indivíduos sem antecedentes de complicações no fígado. ${ }^{11}$ Potencialmente fatal, a FHF pode evoluir rapidamente para falência múltipla de órgãos, ou ainda, para hipertensão intracraniana e edema cerebral em pacientes com encefalopatia hepática severa. ${ }^{12}$ Nos últimos anos, houve uma clara mudança na etiologia da FHF, com a injúria induzida por drogas, aumentando expressivamente nos países ocidentais, em detrimento da etiologia viral. ${ }^{13}$ Estudos norte-americanos têm registrado, recentemente, que o uso e a administração de diferentes medicamentos estão ligados a mais da metade dos casos de FHF. ${ }^{14}$

Relacionadas com a etiologia medicamentosa da FHF estão as drogas envolvidas na TMD da hanseníase (Clofazimina, Rifampicina e Dapsona), recomendadas desde a década de 1980 pela OMS, momento em que a resistência à monoterapia com dapsona passou a ser 
um grande problema no manejo da doença..$^{15}$ Apesar do notado sucesso da terapia, verificou-se a existência de efeitos adversos que acabaram levando à piora do quadro do doente, como acometimento hepático, sabidamente decorrente do uso da rifampicina ou da administração da dapsona na chamada síndrome de hipersensibilidade à dapsona (SHD).

As síndromes de hipersensibilidade são reações idiossincráticas raras à exposição às drogas que, diferentemente das demais reações adversas, podem começar após prolongado contato com a medicação. As drogas que mais frequentemente causam a síndrome incluem anticonvulsivantes, como fenitoína e carbamazepina; antibióticos sulfonamidas e sulfonas. As sulfonamidas, com destaque a dapsona, são bastante conhecidas por causar lesão hepática. A hepatotoxicidade parece ser um efeito característico dessa classe, isso porque, praticamente, todas as sulfonamidas usadas na atualidade foram associadas a casos raros, mas consideráveis, de lesão hepática de etiologia medicamentosa. O padrão de lesão apresenta-se de forma variável, geralmente misto, com acometimento hepatocelular ou colestático. A lesão, na maior parte das vezes, acontece precedida ou acompanhada de sinais de hipersensibilidade, como febre, erupção cutânea, edema facial, linfadenopatia, artralgias e eosinofilia ou linfocitose atípica. ${ }^{16}$

Em contrapartida, a patogênese da SHD não é simples e vários fatores podem estar implicados na sua origem. Um mecanismo proposto envolve a formação de haptenos a partir de metabólitos da dapsona, que subsequentemente produzem anticorpos anti-dapsona, induzindo diferentes respostas adversas. ${ }^{17}$ Esses metabólitos tóxicos são produzidos como resultado do metabolismo oxidativo pelo citocromo $\mathrm{P} 450$, mieloperoxidases e peroxidases da tireóide e, geralmente, são ainda mais biotransformados e desintoxicados pela epóxido-hidroxilase. Pacientes que não possuem essa enzima ou têm mutações disfuncionais em seu gene são mais propensos a desenvolver a síndrome. Metabólitos oxidativos também podem causar a liberação de citocinas que alertam o sistema imunológico do estresse e dano celular. ${ }^{17}$

O paciente em questão apresentou a SHD com quadro de anemia, dermatite esfoliativa, icterícia progressiva, elevação importante das enzimas hepáticas, alargamento do INR e aumento das bilirrubinas. Ao quadro, somou-se o diagnóstico de FHF, já que apresentou encefalopatia hepática severa em adição à queda da função do órgão. Uma vez identificada a gravidade, coube à equipe a definição da medida terapêutica mais adequada para o paciente, bem como a avaliação de uma possível indicação de transplante emergencial de fígado, medida fundamental no manejo do caso.
Nos diferentes sistemas de avaliação da indicação do transplante hepático em casos de FHF medicamentosa, é utilizada uma gama de variáveis que podem contribuir na decisão, como etiologia, intervalo de tempo icteríciaencefalopatia e a idade do paciente, bem como dados laboratoriais, como níveis séricos de fator $\mathrm{V}$, lactato, bilirrubinas e INR. Tais parâmetros organizam-se em diferentes sistemas de avaliação de prognóstico e indicação de TOF. ${ }^{18}$

Entre esses sistemas, com importante valor de predição de prognóstico em casos de FHF, o critério do hospital do King's College (formulado em 1989) é um fundamental preditor de prognóstico. Isso acontece, pois relaciona prognósticos pobres e, portanto, a necessidade de TOF em pacientes com a presença de tempo de protrombina maior que 100 s ou com a associação de três dentre os seguintes cinco fatores de risco: idade menor que 10 ou maior que 40 anos; etiologia relacionada à reação a drogas (não relacionada com Hepatite A ou B); duração da icterícia até aparecimento de encefalopatia hepática $>7$ dias; tempo de protrombina $>50$ s; e bilirrubina sérica $>300 \mu \mathrm{mol} / \mathrm{L}^{19}$

Preenchendo os critérios do King's College, com um score MELD de 42 e um quadro clínico grave, o paciente foi listado para transplante hepático. Transplantado através da técnica cirúrgica piggyback, com enxerto de doador falecido, evoluiu com melhora substancial e progressiva no pós-operatório. Através dessa medida terapêutica implementada pela equipe, o valor e o potencial do emprego do TOF em determinados casos de FHF relacionada às reações de hipersensibilidade a drogas, crescem tanto em experiência quanto em relação a apresentação de resultados.

$\mathrm{Na}$ evolução do tratamento da FHF, o TOF surge, dessa forma, como um significativo recurso, sendo capaz de amenizar o cenário delicado e o prognóstico pobre desse acometimento. Na era pré-transplante, apenas $20 \%$ dos pacientes em FHF sobreviviam. Atualmente, com a evolução dessa técnica cirúrgica, melhores cuidados no manejo intensivo e introdução de drogas imunossupressoras mais seletivas, ele oferece sobrevida em torno de $65 \%$ e, em algumas séries, chegando a mais de $80 \%$, ficando claro que esse pode ser um caminho inovador na terapêutica atual e futura. ${ }^{20}$

O estudo estatístico foi efetuado através do software informático IBM SPSS Statistics $®$ V.26. Para a análise descritiva foram apresentadas médias e desviospadrão das variáveis contínuas com simetria das distribuições; no caso das distribuições assimétricas, foi utilizada a mediana, com menção dos valores mínimos e máximos. 


\section{CONCLUSÃO}

O conhecimento dos efeitos colaterais das drogas envolvidas no tratamento da hanseníase é de fundamental importância, principalmente em países com alta prevalência da doença, a fim de que haja um protocolo terapêutico mais específico e eficiente. A SHD é uma complicação grave e rara da utilização da droga, que pode levar à FHF, necessitando de uma intervenção emergencial da equipe médica.
Nesse contexto, o transplante hepático surge como uma alternativa terapêutica que parece ser segura e eficaz quando indicado para pacientes com FHF medicamentosa, como no caso descrito. Fica clara, assim, a importância da análise e da evolução clínica detalhadas de pacientes em tratamento para a hanseníase, com o intuito de abordar de forma precoce os possíveis efeitos adversos.

\section{ABSTRACT}

The incidence of drug-induced acute liver failure has increased. Among the drugs involved are especially antibiotics such as rifampicin and dapsone involved in the treatment of leprosy. Dapsone hypersensitivity syndrome (DHS) is a rare adverse reaction. This syndrome is characterized by fever, exfoliative dermatitis, lymphadenopathy, anemia and liver involvement, which includes hepatocyte necrosis and cholestasis. Acute liver failure due to DHS may be an indication for emergency liver transplantation, a rarely reported condition in the literature. For the present study, the patient's pre and postoperative data were analyzed through his medical record. A non-systematic search was conducted by using PubMed and Scielo database to find the most up-to-date literature on drug-induced acute liver failure and DHS. A 23-year-old male patient was admitted to a referral center after undergoing combined therapy for Virchowian leprosy (dapsone, rifampicin, clofazimine) for approximately 30 days. The patient presented severe progressive jaundice, anemia, coagulopathy and grade IV encephalopathy (West-Haven criteria) in addition to elevated liver enzymes and acute kidney injury. The patient was enlisted for liver transplantation with 40 MELD score (model for end-stage liver disease). The surgical technique employed was piggyback using a deceased donor allograft (donation after brain death). The post-transplant clinical improvement was substantial. Histopathological assessment of the native liver showed compatibility with drug-induced acute hepatitis. Although rarely indicated in cases of acute hepatitis associated to DHS, liver transplantation has been shown to be effective in this context, improving the prognosis of otherwise terminal patients. The present case should alert for the need of clinical and biochemical monitoring of patients undergoing treatment for leprosy. Patients and professionals should also be informed on DHS signs and symptoms, in view of its potentially fatal repercussions.

Keywords: Liver Failure, Acute; Chemical and Drug Induced Liver Injury, Dapsone, Leprosy, Liver Transplantation

\section{AGRADECIMENTOS}

Agradecemos à equipe multidisciplinar da Unidade de Transplante de

Fígado de Pernambuco, que se dedica continuamente e de maneira inspiradora ao cuidado dos pacientes, incluindo o do caso apresentado. 


\section{REFERÊNCIAS}

1. Eichelmann K, González SE, Salas-Alanis JC, OcampoCandiani J. Lepra: puesta al día. Definición, patogénesis, clasificación, diagnóstico y tratamiento. Actas DermoSifiliográficas. 2013;104(7):554-63.

2. World Health Organisation. Global leprosy update, 2017: reducing the disease burden due to leprosy. Weekly Epidemiological Record. 2018;93(35):445-56.

3. Deps PD, Nasser S, Guerra P, Simon M, Birshner Rde C, Rodrigues LC. Adverse effects from multi-drug therapy in leprosy: a Brazilian study. Leprosy Review. 2007;78(3):21622.

4. Nair SP. A 19-Year Retrospective Study of Adverse Drug Reactions to Multidrug Therapy in Leprosy Requiring a Change in Regime. Indian Dermatology Online Journal. 2018;9(1):33-6. doi: 10.4103/idoj.IDOJ_116_17.

5. Lorenz M, Wozel G, Schmitt J. Hypersensitivity reactions to dapsone: a systematic review. Acta Derm Venereol. 2012;92(2):194-9. doi: 10.2340/00015555-1268.

6. Semaan G Kosseifim, Bhuvana Guha, Dima N Nassour, David S Chi, Guha Krishnaswamy. The Dapsone Hypersensitivity Syndrome revisited: a potentially fatal multisystem disorder with prominent hepatopulmonary manifestations. J Occup Med Toxicol. 2006;1:9. doi: 10.1186/1745-6673-1-9

7. Rao PN, Lakshmi TS. Increase in the incidence of dapsone hypersensitivity syndrome-an appraisal. Leprosy Review. 2001;72(1):57-62.

8. Tian W, Shen J, Zhou M, Yan L, Zhang G. Dapsone hypersensitivity syndrome among leprosy patients in China. Leprosy Review. 2012;83(4):370-7

9. Garcia A, Yi NJ, Lee KB, Lee JM, Choi YR, Suh SW, et al. Fulminant hepatitis linked to dapsone hypersensitivity syndrome requiring urgent living donor liver transplantation: a case report. Pediatr Transplant. 2014;18(7):E240-5. doi: 10.1111/petr.12343.

10. Olivo R, Guarrera JV, Pyrsopoulos NT. Liver Transplantation for Acute Liver Failure. Clinics in Liver Disease. 2018;22(2):409-17. doi: 10.1016/j.cld.2018.01.014.
11. Lee WM, Stravitz RT, Larson AM. Introduction to the revised American Association for the Study of Liver Diseases position paper on acute liver failure 2011. Hepatology. 2012;55(3):965-7.

12. Bernal W, Hyyrylainen A, Gera A, Audimoolam VK, Mcphail MJ, Auzinger G, et al. Lessons from look-back in acute liver failure? A single centre experience of 3300 patients. Journal Of Hepatology. 2013;59(1):74-80. doi: 10.1016/j.jhep.2013.02.010.

13. Merritt WT. Perioperative concerns in acute liver failure. Int Anesthesiol Clin 2006;44(4):37-57.

14. Tujios SR, Lee WM. Acute liver failure induced by idiosyncratic reaction to drugs: Challenges in diagnosis and therapy. Liver International. 2017;38(1):6-14,. doi: 10.1111/liv.13535.

15. Schreuder PA, Noto S, Richardus, JH. Epidemiologic trends of leprosy for the 21st century. Clinics In Dermatology. 2016;34(1):24-31. doi: 10.1016/j. clindermatol.2015.11.001.

16. LiverTox: Clinical and Research Information on Drug-Induced Liver Injury [Internet]. Bethesda (MD): National Institute of Diabetes and Digestive and Kidney Diseases; 2012-2017 Dec 5.

17. Kinehara, Y, Kijima, T, Inoue, K, Hirata, H, Takeuchi, Y, Fukushima, K, et al. (2015). Dapsone hypersensitivity syndrome-related lung injury without eosinophilia in the bronchoalveolar lavage fluid. Internal medicine. 2015;54(7):827-31

18. O'Grady, J. Timing and benefit of liver transplantation in acute liver failure. Journal Of Hepatology. 2014;60(3):p.663-670, doi: 10.1016/j.jhep.2013.10.024.

19. O'Grady J, Alexander GJ, Hayllar KM, Williams R. Early indicators of prognosis in fulminant hepatic failure. Gastroenterology. 1989;97:439-45

20. Castaldo ET, Chari RS. Liver transplantation for acute hepatic failure. HPB. 2006;8:29-34. 